\title{
The Definition of Complex Uncertainties in B- spline Surface by using Normal Type-2 Triangular Fuzzy Number
}

\author{
Reedzal Shahreen Adesah* and Rozaimi Zakaria \\ ${ }^{I}$ Mathematics, Graphics and Visualization Research Group (MGRAVS), Faculty of Science and Natural \\ Resources, Universiti Malaysia Sabah, 88400 Kota Kinabalu, Sabah, Malaysia
}

\begin{abstract}
In this paper, Normal Type-2 Triangular Fuzzy Number (NT2TFN) is used for defining complex uncertainties data to construct an approximation of B-spline surface. The type-2 fuzzy set is used to define the complex uncertainties due to type-1 fuzzy has limited efficiency to define the complex uncertainties problem.NT2TFN is based on the concept of Normal Type-2 Fuzzy Set Theory (NT2FST) and the Type-2 Fuzzy Number (T2FN). NT2TFNis used to define the complex uncertainties data before the three technique were implemented. These techniques include the fuzzification process by using alpha-cut operation with two determined value of alpha which is 0.5 and 0.8 , the type-reduction process and the defuzzification process. Therefore, the finalize model of Normal Type-2Fuzzy B-spline Surface (NT2FBsS) for two determined value of alpha can be achieved and a new implementation to construct a geometry model by using NT2TFN on defining complex uncertainty data to fuse with Bspline surface is accomplished.
\end{abstract}

Keywords: Normal Type-2 Fuzzy Set Theory, Fuzzy B-spline surface, Normal Type-2 Fuzzification, Type-reduction, Defuzzification

\section{INTRODUCTION}

Zadeh has introduced Type-2 Fuzzy Set Theory (T2FST) ten years after Fuzzy Set Theory (FST) or Type-1 Fuzzy Set Theory (T1FST) has been introduced in 1965 (Zadeh, 1965; 1975). T2FST is used to define complex uncertainty problems which ordinary fuzzy set theory cannot handle. Complex uncertainty happens when the uncertainty becomes more uncertainty, or the problems have a higher level of uncertainty than the usual uncertainty problem. Karnik and Mendel (2001) make T2FST easier to use and understand after explaining it briefly and T2FST is widely used in many fields for defining complex uncertainty problems (Adesah \& Zakaria, 2017).

There are two special cases on T2FST (Zakaria \& Wahab, 2013). The first case is when complex uncertainty is on the footprints where type-2 membership function values are in specific value and another case is when complex uncertainty is on the type-2 membership function where footprints are in the single value. For the first case, Hamrawi and Coupland have introduced types of T2FST which is normal and perfectly-normal. Perfectlynormal T2FST happens when both Lower Membership Function (LMF) and Upper Membership Function (UMF) is normal while NT2FST happens when only UMF is normal (Hamrawi \& Coupland, 2009). Besides, for the second case, there is only one type of T2FST which is introduced by Zakaria and Wahab in 2013.

Normal Type-2 Fuzzy Set Theory (NT2FST)is used in this paper to define complex uncertainties in a set of data which is applied in B-spline surface function to build a Normal Type-2 Fuzzy B-spline Surface (NT2FBsS). We

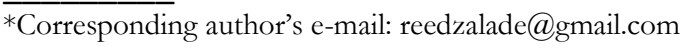


will discuss the fuzzification process by using alpha-cut operation with two determined value of alpha which is 0.5 and 0.8 . This is because NT2FST gives two situations for the alpha-cut operation which the alpha-cut will go through towards all and some of NT2TFN. The alpha-cut operation is held before the process of type-reduction takes over to reduce T2FST to T1FST. Then, the process of defuzzification is implemented to obtain the finalize model of NT2FBsS.

This paper constructed as follows: Section II discusses the basic definition of NT2FST to define complex uncertainty data points and all the three techniques include the fuzzification process, the type-reduction process and the defuzzification process. Then, Section III discusses the formation of NT2FBsS. The conclusion of this method is explained in the next section.

\section{MATERIALS AND METHODS}

First, the complex uncertainties data is defined with the definition of T2FST in Definition 2.1 and Type-2 Fuzzy Number (T2FN) in Definition 2.2 which T2FN is defined as Normal Type-2 Triangular Fuzzy Number (NT2TFN) as we can see through Figure 1 (Adesah \& Zakaria, 2017). Then, after we define the complex uncertainties data, the process will continue with the fuzzification process, the type-2 reduction process and the defuzzification process. All definition of these processes is presented in this section for both two determined value of alpha (Adesah et al., 2017).

Definition 2.1. $\overline{\overline{F S}}$ is stand for Type-2 Fuzzy Sets (T2FS), is described by a type-2 membership function $\mu_{\overline{\overline{F S}}}(x, u)$, where $x \in X$ and $u \in U_{x} \subseteq[0,1]$ that is,

$$
\overline{\overline{F s}}=\left\{(x, u), \mu_{\overline{F S}}(x, u) \mid \forall x \in X, \forall u \in U_{x} \subseteq[0,1]\right\}
$$

where $0 \leq \mu_{\overline{F S}}(x, u) \leq 1$ (Adesah \& Zakaria, 2017).

Definition 2.2. $\mathrm{T} 2 \mathrm{FN}$ is described as a T2FS which has a numerical domain. The following four constraints are used to define an interval T2FS, where $\overline{\overline{F S}}_{\alpha}=\left\{\left[p^{\alpha}, q^{\alpha}\right],\left[r^{\alpha}, s^{\alpha}\right]\right\}$, $\forall \alpha \in[\mathbf{0}, 1], \forall p^{\alpha}, q^{\alpha}, r^{\alpha}, s^{\alpha} \in \square \quad$ (Figure 1): i. $\quad p^{\alpha} \leq q^{\alpha} \leq r^{\alpha} \leq s^{\alpha}$.

ii. $\left[p^{\alpha}, s^{\alpha}\right]$ and $\left[q^{\alpha}, r^{\alpha}\right]$ develop a function that is convex and $\left[p^{\alpha}, s^{\alpha}\right]$ develop a function is normal.

iii. $\forall \alpha_{1}, \alpha_{2} \in[0,1]:\left(\alpha_{2}>\alpha_{1}\right) \Rightarrow\left(\left[p^{\alpha_{1}}, r^{\alpha_{1}}\right] \supset\left[p^{\alpha_{2}}, r^{\alpha_{2}}\right]\right.$, $\left.\left[q^{\alpha_{1}}, s^{\alpha_{1}}\right] \supset\left[q^{\alpha_{2}}, s^{\alpha_{2}}\right]\right)$, for $r^{\alpha_{2}} \geq q^{\alpha_{2}}$

iv. If the maximum of the membership function developed by $\left[q^{\alpha}, r^{\alpha}\right]$ is the level $\alpha_{\Upsilon}$, which is, $\left[q^{\alpha_{k}}, r^{\alpha_{k}}\right]$, then $\left[q^{\alpha_{k}}, r^{\alpha_{k}}\right] \subset\left[p^{\alpha=1}, s^{\alpha=1}\right]$ (Aguero \& Vargas, 2007)

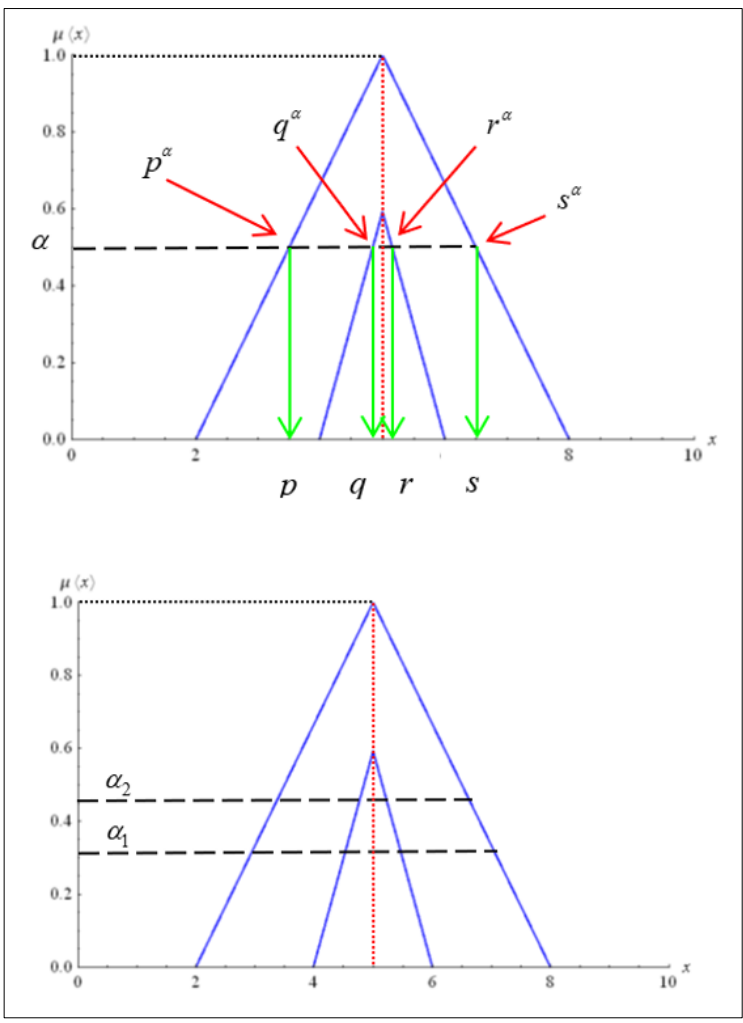

Figure 1. The interpretation of an interval T2FN.

Next, the fuzzification process is applied towards NT2TFN.After the complex uncertainties data is defined as Figure 1, the alpha-cut operation is applied to the data. The operation applied gives two cases of alpha values by using Definition 2.3 and 2.4.

Definition 2.3. It is given $\overline{\overline{F S}}$ that is $\mathrm{T} 2 \mathrm{FN}$, which 
$\mathrm{H}\left(\overline{\overline{F s_{\uparrow}}}\right)$ and $\mathrm{H}\left(\overline{\overline{F s_{\downarrow}}}\right)$ are the height for Upper Membership Function (UMF) and Lower Membership Function (LMF) respectively, then T2FN is called NT2TFN if $\mathrm{H}\left(\overline{\overline{F s_{\downarrow}}}\right)<\mathrm{H}\left(\overline{\overline{F s_{\uparrow}}}\right)=\mathbf{1}$. This definition can be interpreted in Figure 2.

Then, the alpha-cut operation is applied towards NT2TFN in Definition 2.4 (Adesah \& Zakaria, 2017).

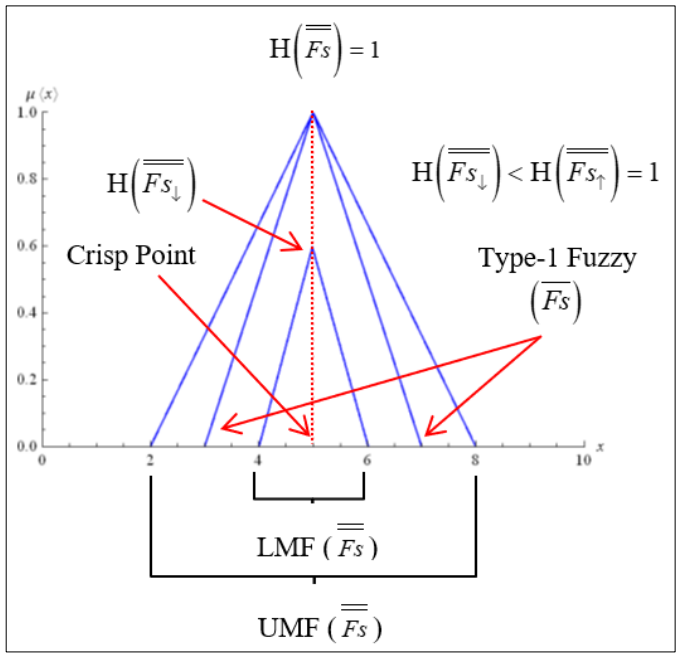

Figure 2. The interpretation of NT2TFN.

Definition 2.4. Let $\overline{\overline{F S}}$ is denoted as NT2TFN with $\overline{\overline{F S}}_{(i, j)} \in \overline{\overline{F S}} \quad$ where $\quad i=0,1, \ldots, n-1 \quad$ and $j=0,1, \ldots, m-1$. Then, $\stackrel{N=}{\overline{F s_{\alpha}}}$ is the alpha-cut operation of NT2TFN for $\alpha_{j}<\alpha_{j}^{\downarrow}=\mathrm{H}(\overline{\overline{F S}} \downarrow) \leq \alpha_{j}^{\uparrow}=\mathrm{H}(\overline{\overline{F S}} \uparrow)$ which is given as equation as follows(Figure 3) $[9,10]$.

$$
\begin{aligned}
& N \overline{\overline{F s}}_{i_{\alpha_{j}=\alpha_{j}<\alpha_{j} \leq \alpha_{j}^{\uparrow}}}=\left\langle\overline{\overline{F s}}{\overleftarrow{i_{\alpha_{j}}}}^{\leftarrow}, F s_{i}, \overline{\overline{F s}}{\overrightarrow{i_{\alpha_{j}}}}\right\rangle
\end{aligned}
$$

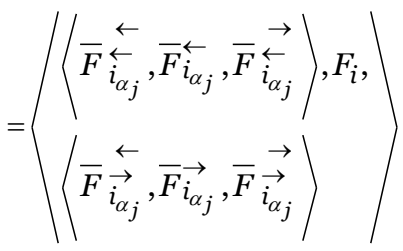

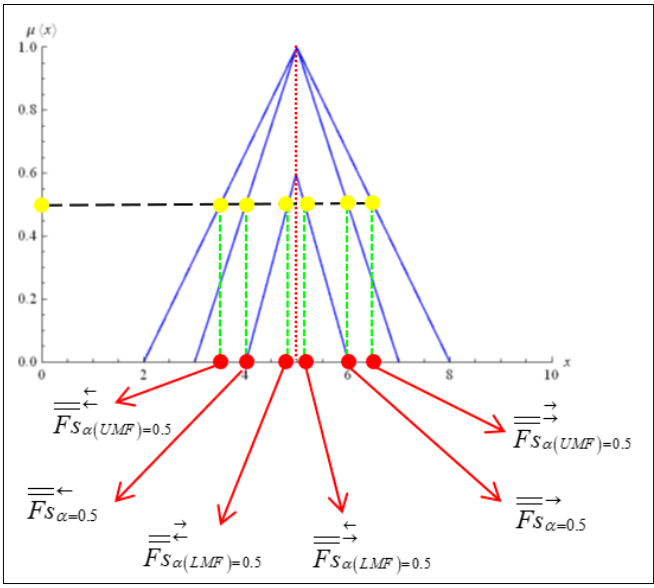

Figure 3. The operation of alpha-cut towards NT2TFN with $\alpha_{j}<\alpha_{j}^{\downarrow} \leq \alpha_{j}^{\uparrow}$.

However, when $\alpha_{j}^{\downarrow}<\alpha_{j} \leq \alpha_{j}^{\uparrow}$ for the alpha-cut operation of NT2TFN, then the definition become as follow (Figure 4).

$$
\begin{aligned}
& N \overline{\overline{F s}}_{i_{\alpha_{j}=\alpha_{j}<\alpha_{j} \leq \alpha_{j}}}=\left\langle\overline{\overline{F s}}{\overleftarrow{i_{\alpha_{j}}}}, F s_{i}, \overline{\overline{F s}} \overrightarrow{i_{\alpha_{j}}}\right\rangle \\
& =\left\langle\begin{array}{l}
\left\langle\bar{F}_{i_{\alpha_{j}}}^{\leftarrow}, \bar{F}_{i_{\alpha_{j}}}, \mathrm{o}\right\rangle, F_{i}, \\
\left.\mathrm{o}, \bar{F}_{i_{\alpha_{j}}}, \bar{F} \overrightarrow{i_{\alpha_{j}}}\right\rangle
\end{array}\right\rangle
\end{aligned}
$$

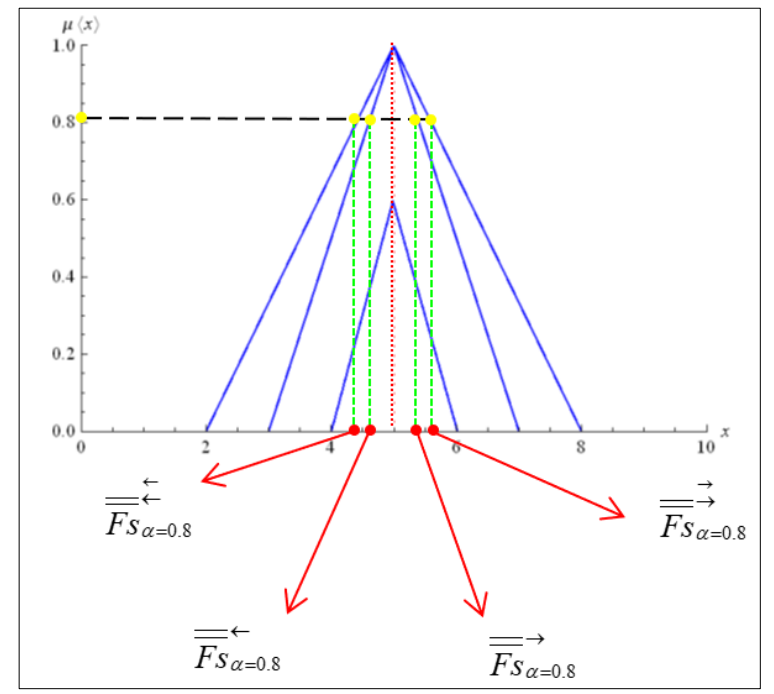

Figure 4. The operation of alpha-cut towards NT2TFN with $\alpha_{j}^{\downarrow}<\alpha_{j} \leq \alpha_{j}^{\uparrow}$.

The fuzzification process applies to two cases of alpha values which is $\alpha_{j}<\alpha_{j}^{\downarrow} \leq \alpha_{j}^{\uparrow}$ and $\alpha_{j}^{\downarrow}<\alpha_{j} \leq \alpha_{j}^{\uparrow}$. For the case of $\alpha_{j}<\alpha_{j}^{\downarrow} \leq \alpha_{j}^{\uparrow}, 0.5$ is determined to be the alpha- 
cut value. It is because 0.5 is in the range of $\alpha_{j}<\alpha_{j}^{\downarrow} \leq \alpha_{j}^{\uparrow}$. Same goes to the case of $\alpha_{j}^{\downarrow}<\alpha_{j} \leq \alpha_{j}^{\uparrow}$, 0.8 is determined to be the alpha-cut value which 0.8 is in the range of $\alpha_{j}^{\downarrow}<\alpha_{j} \leq \alpha_{j}^{\uparrow}$.

Next, after NT2TFN went through the fuzzification process, the type-reduction process is applied to reduce the type-2 fuzzy model to type-1 fuzzy model. This will allow the application of the defuzzification process to take over. The type-reduction process is applied for both cases of alpha values for $\alpha_{j}<\alpha_{j}^{\downarrow} \leq \alpha_{j}^{\uparrow}$ and $\alpha_{j}^{\downarrow}<\alpha_{j} \leq \alpha_{j}^{\uparrow}$. Definition 2.5 is implemented in both cases.

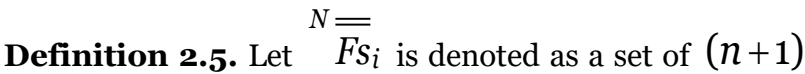
NT2TFN after the alpha-cut operation, then the method of type-reduction $\alpha$-T2TFN, $\quad \stackrel{N \overline{F s}}{i_{\alpha}}$ is defined by

$$
N \overline{F s}_{\alpha}=\left\{\begin{array}{l}
N \overline{F s}_{i_{\alpha_{j}}}=\left\langle\overline{F s_{\alpha_{j}}}, F s_{i}, \overrightarrow{F s_{\alpha_{j}}}\right\rangle \\
\mid i=0,1, \ldots, n ; j=1,2, \ldots, m
\end{array}\right\}
$$

where $\overline{F s_{i_{\alpha_{j}}}} \leftarrow$ and $\overline{F s_{\alpha_{\alpha_{j}}}}$ are left and right type-reduction of alpha-cut NT2TFN. Definition for both left and right type-reduction become as follow (Zakaria et al., 2013).

$$
\begin{aligned}
& N \overline{F s}_{\alpha}=\left\langle\overline{F s}_{i_{\alpha_{j}}}^{\leftarrow}, F s_{i}, \overrightarrow{F s_{i_{j}}}\right\rangle
\end{aligned}
$$

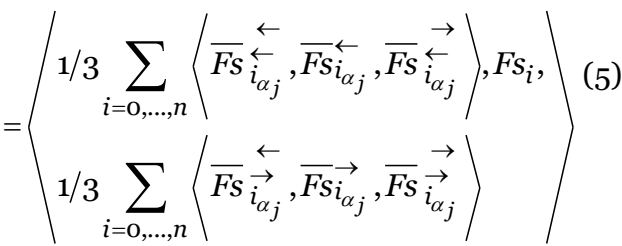

Lastly, to obtain the finalize model, the process of defuzzification is implemented after the type-reduction process is applied. Definition 2.6 is also used in both cases.

Definition 2.6. Let ${ }^{N} \overline{F \boldsymbol{S}}_{\alpha}$ is denoted as type-reduction of NT2TFN, then ${ }^{N} F s_{\alpha}$ named as defuzzification of NT2TFN for ${ }^{N} \overline{F s}_{i_{\alpha}}$ if for every ${ }^{N} \overline{F s}_{i_{\alpha}} \in{ }^{N} \overline{F s}_{i}$,

$$
F s=\left\{F s_{i_{\alpha_{j}}}\right\} \text { for } i=0,1, \ldots, n ; j=1,2, \ldots, m
$$

with $F s=\sum_{\substack{i=0, \ldots, n ; \\ j=1, \ldots, m}}\left\langle\overline{F s}_{i_{\alpha_{j}}}^{\leftarrow}, F s_{i}, \overline{F s}_{i_{\alpha_{j}}}\right\rangle / 2 j+1$ (Wahab \&

Zakaria, 2013).

\section{RESULTS AND DISCUSSION}

After defining the complex uncertainties data is finished by using NT2TST, NT2FBsS is constructed with the implementation of NT2TFN with processes of fuzzification, type-reduction and defuzzification as mentioned before. All definition 2.1 until 2.3 is blended with the control points of B-spline surface function denoted by $\overline{\overline{F s}}_{i, j}$, which is given as follows.

$$
\begin{gathered}
\overline{\overline{F B s S}}(u, v)=\sum_{i=0}^{n} \sum_{j=0}^{m} \overline{\overline{F s}} i, j N_{i, k}(u) N_{j, l}(v) \\
\text { with } 0 \leq u, v \leq 1
\end{gathered}
$$

with $N_{i, k}(u)$ and $N_{j, l}(v)$ are B-spline functions and $\overline{\overline{F s}}_{i, j}$ is three dimension fuzzy control points with $i=0,1, \ldots, n-1 \quad$ and $j=0,1, \ldots, m-1$. These can be illustrated through Figure 5(Zakaria et al., 2014).

Then, the processes of fuzzification, reduction and defuzzification are implemented by using definition 2.4 until 2.6 towards B-spline surface function for both cases of alpha values for $\alpha_{j}<\alpha_{j}^{\downarrow} \leq \alpha_{j}^{\uparrow}$ and $\alpha_{j}^{\downarrow}<\alpha_{j} \leq \alpha_{j}^{\uparrow}$ interpreted in Table 1 and Table 2 respectively with the definition of NT2TFN which blended with the control points of B-spline.

As we can see in Table 1 and Table 2, the surface obtained in Table 2 is closer to the crisp model which is a grey coloured surface. This is because the 0.8 is used for the alpha-cut value closer to 1 which is the value for the crisp model. Furthermore, there are just five surfaces in Table 2 instead of seven surfaces in Table 1. This is because the alpha-cut value of 0.5 in Table 1 will go through towards all NT2TFN and the alpha-cut value of o.8 in Table 2 did not go through towards UMF. 


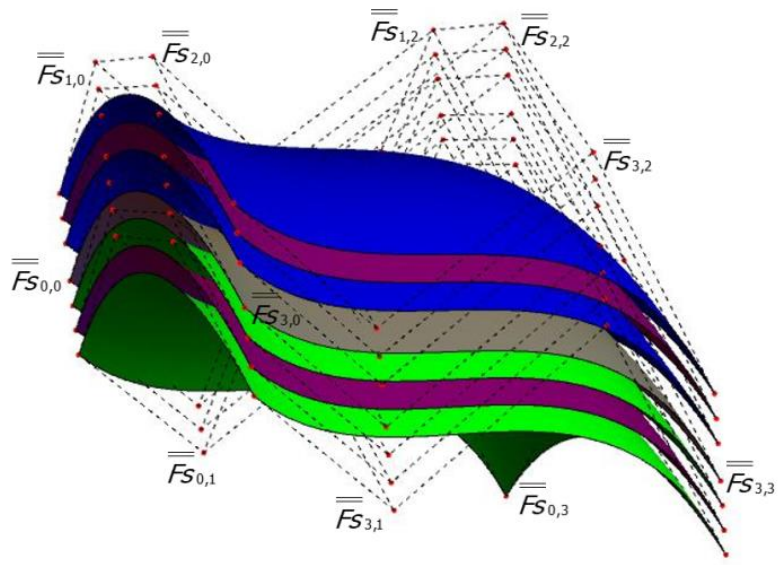

(a)

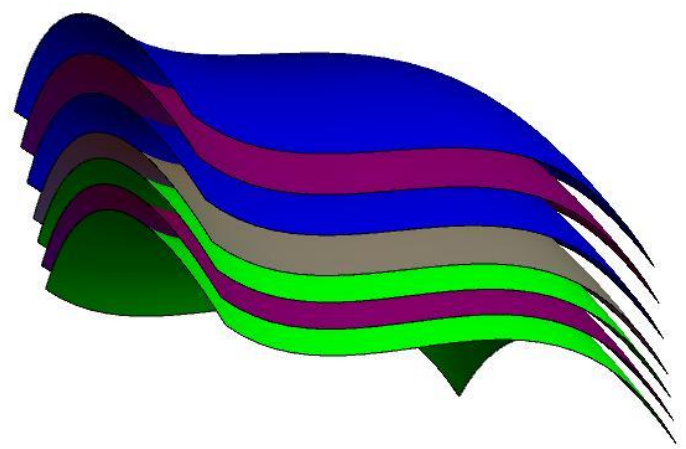

(b)

Figure 5. Model of fuzzified NT2FBsS with control points in (a) and without control points in (b).

Table 1. Model of fuzzification, reduction and defuzzification of NT2FBsSforthe case of alpha values for $\alpha_{j}<\alpha_{j}^{\downarrow} \leq \alpha_{j}^{\uparrow}$ with the definition.

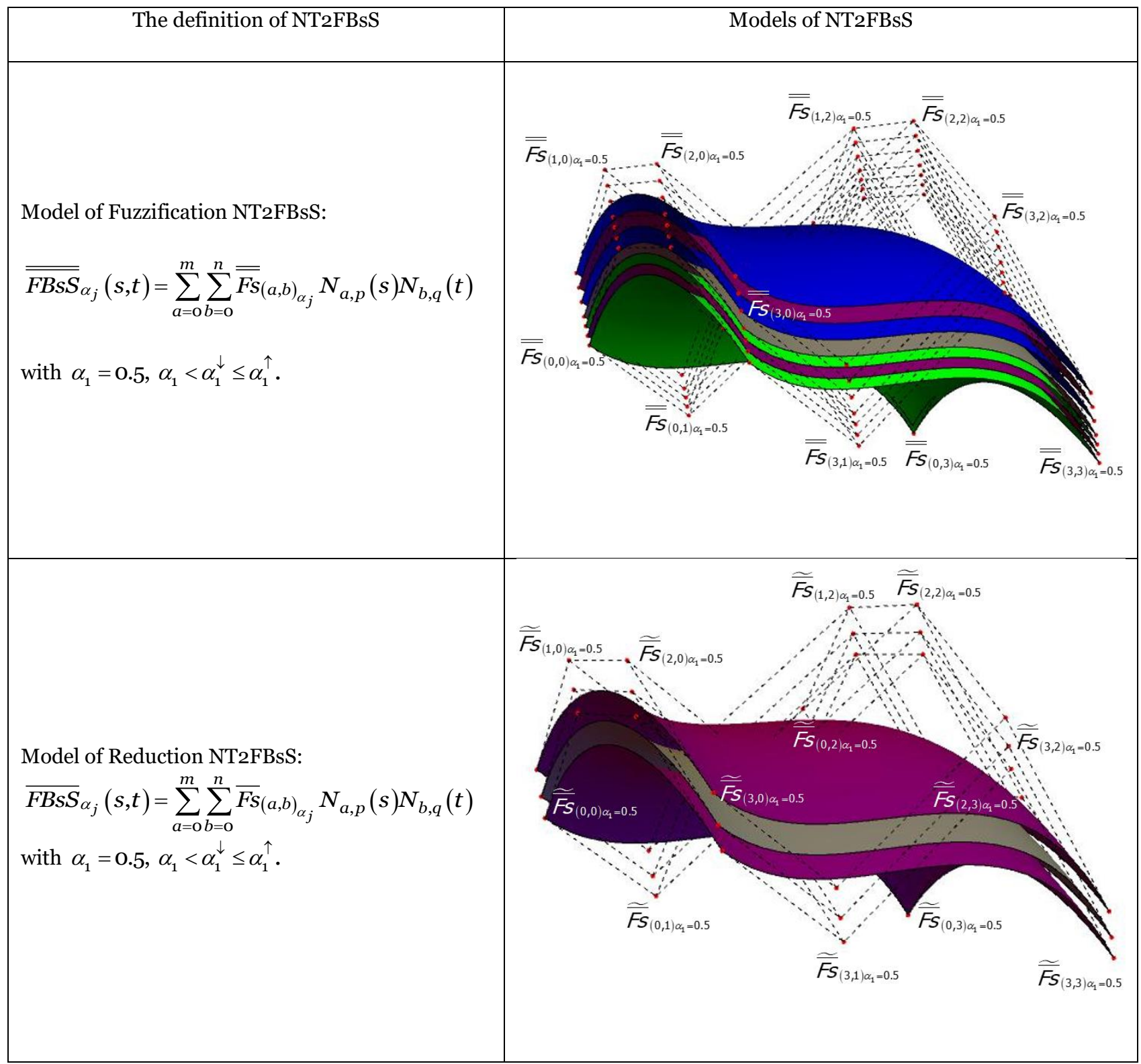


Model of Defuzzification NT2FBsS:

$F B s S_{\alpha_{j}}(s, t)=\sum_{a=\mathrm{o}}^{m} \sum_{b=\mathrm{O}}^{n} F s_{(a, b)_{\alpha_{j}}} N_{a, p}(s) N_{b, q}(t)$ with $\alpha_{1}=0.5, \alpha_{1}<\alpha_{1}^{\downarrow} \leq \alpha_{1}^{\uparrow} . F_{i, j}$ is a crisp model.

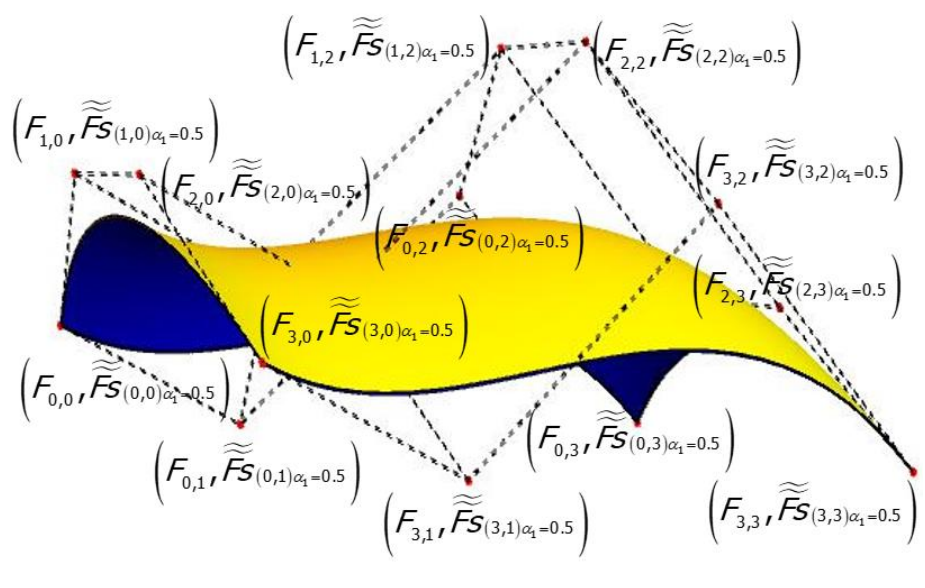

Table 2. Model of fuzzification, reduction and defuzzification of NT2FBsSforthe case of alpha values for $\alpha_{j}^{\downarrow}<\alpha_{j} \leq \alpha_{j}^{\uparrow}$ with the definition.

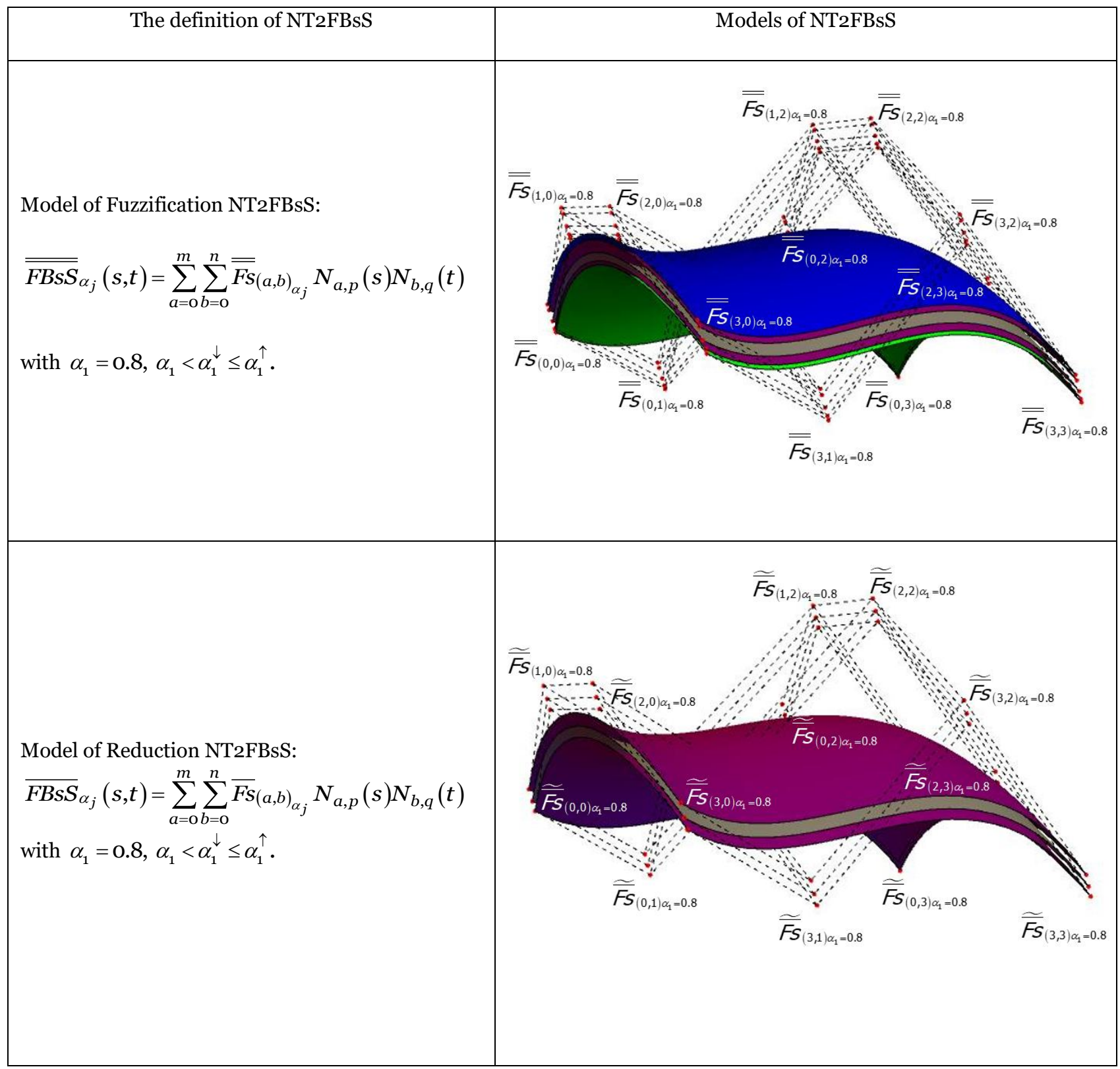


Model of Defuzzification NT2FBsS:

$F B s S_{\alpha_{j}}(s, t)=\sum_{a=\mathrm{o}}^{m} \sum_{b=\mathrm{O}}^{n} F s_{(a, b)_{\alpha_{j}}} N_{a, p}(s) N_{b, q}(t)$ with $\alpha_{1}=0.8, \alpha_{1}<\alpha_{1}^{\downarrow} \leq \alpha_{1}^{\uparrow} . F_{i, j}$ is a crisp model.

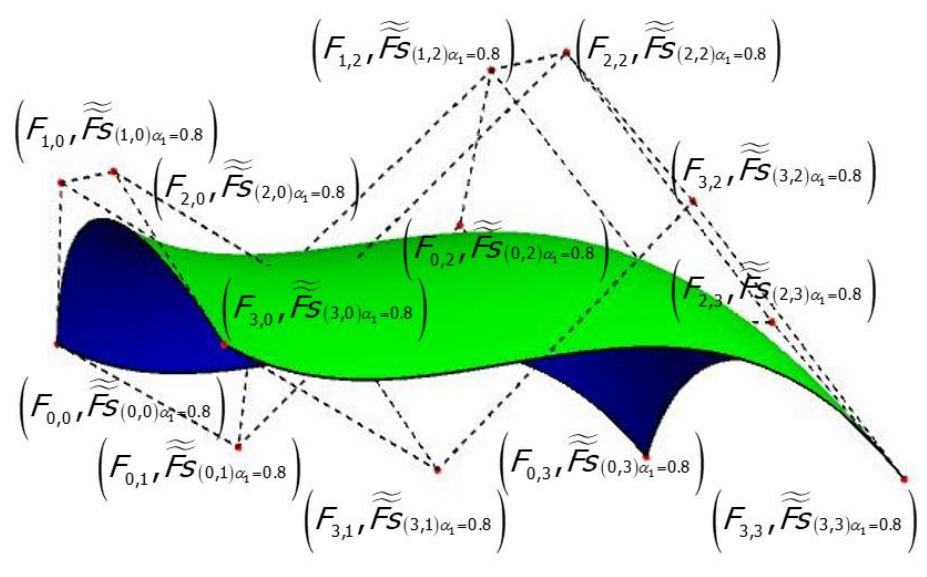

\section{CONCLUSION}

In this paper, we proposed a new implementation to construct a geometry model by using normal T2FST instead of perfectly normal T2FST to fuse with B-spline function which to handle complex uncertainty of data. The model NT2FBsS has been constructed by using the definition of T2FST in the form of NT2TFN to handle complex uncertainties in a set of data and being blended in function of B-spline surface. For further studies of this model, it can be extended with other functions such as Bezier surface and rational B-spline surface. Furthermore, the method of complex uncertainty is on the type-2 membership function where footprints are in the single value can be implemented to construct a curve or surface model.

\section{ACKNOWLEDGEMENTS}

The authors would like to express sincere gratitude to Mathematics, Graphics and Visualization Research Group (M-GRAVS), Faculty of Science and Natural Resources of University of Malaysia Sabah (UMS) and Ministry of Higher Education (MOHE) for funding (RAGoo62SG2015)and providing facilities to accomplish this research. 


\section{REFERENCES}

Adesah, R.S. and Zakaria, R., 2017, September. Normal Type-2 Fuzzy Geometric Curve Modeling: A Literature Review. In Journal of Physics: Conference Series (Vol. 890, No. 1, p. 012087). IOP Publishing.

Adesah, R.S., Zakaria, R., Wahab, A.F. and Talibe, A., 2017, September. Type-2 Fuzzy Curve Model. In Journal of Physics: Conference Series (Vol. 890, No. 1, p. 012088). IOP Publishing.

Aguero, J.R. and Vargas, A., 2007. Calculating functions of interval type-2 fuzzy numbers for fault current analysis. IEEE Transactions on Fuzzy systems, 15(1), pp.31-40.

Hamrawi, H. and Coupland, S., 2009, January. Type-2 fuzzy arithmetic using alpha-planes. Proceedings of the IFSAEUSFLAT Conference.

Karnik, N.N. and Mendel, J.M., 2001. Operations on type-2 fuzzy sets. Fuzzy sets and systems, 122(2), pp.327-348.

Wahab, A.F. and Zakaria, R., 2013. Generalized Normal Type-2 Triangular Fuzzy Number. Applied Mathematical Sciences, 7(45), pp.2239-2252.

Zadeh, L.A., 1965. Information and control. Fuzzy sets, 8(3), pp.338-353.

Zadeh, L.A., 1975. The concept of a linguistic variable and its application to approximate reasoning-I. Information sciences, 8(3), pp.199-249.

Zakaria, R. and Wahab, A.F., 2013. On defining complex uncertainty data points by type-2 fuzzy number: two specials cases. Int. Journal of Math Analysis, 7(26), pp.1285-1300.

Zakaria, R., Wahab, A. and Gobithaasan, R.U., 2014. Fuzzy B-Spline surface modeling. Journal of Applied Mathematics, 2014.

Zakaria, R., Wahab, A.F. and Gobithaasan, R.U., 2013, April. Type-2 fuzzy Bezier curve modeling. In AIP Conference Proceedings (Vol. 1522, No. 1, pp. 945-952). AIP. 